\title{
A comparison between statistical properties of solar X-ray flares and avalanche predictions in cellular automata statistical flare models
}

\author{
M. K. Georgoulis ${ }^{1,2}$, N. Vilmer ${ }^{3}$, and N. B. Crosby ${ }^{3,4}$ \\ 1 Section of Astrophysics, Astronomy and Mechanics, Department of Physics, University of Thessaloniki, \\ 54006 Thessaloniki, Greece \\ 2 Johns Hopkins University, Applied Physics Laboratory, 11100 Johns Hopkins Rd., Laurel, MD 20723, USA \\ 3 LPSH, URA 2080 CNRS, DASOP Observatoire de Paris, Section d'Astrophysique de Meudon, 5 place J. Janssen, \\ 92195 Meudon Cedex, France \\ 4 LPCE, UPR 4010 CNRS, 3A, Avenue de la Recherche Scientifique, 45071 Orléans la Source Cedex 2, France \\ Received 20 September 2000 / Accepted 30 November 2000
}

\begin{abstract}
We perform a tentative comparison between the statistical properties of cellular automata statistical flare models including a highly variable non-linear external driver and the respective properties of the WATCH flare data base, constructed during the maximum of solar cycle 21. The model is based on the concept of SelfOrganized Criticality (SOC). The frequency distributions built on the measured X-ray flare parameters show the following characteristics: (1) The measured parameters (total counts, peak count-rates and, to a lesser extent, total duration) are found to be correlated to each other. Overall distribution functions of the first two parameters are robust power laws extending over several decades. The total duration distribution function is represented by either two power laws or a power law with an exponential roll-over. (2) By sub-grouping the peak count-rate and the total counts as functions of duration and constructing frequency distributions on these sub-groups, it is found that the slope systematically decreases with increasing duration. (3) No correlation is found between the elapsed time interval between successive bursts arising from the same active region and the peak intensity of the flare. Despite the inherent weaknesses of the SOC models to simulate realistically a number of physical processes thought to be at work in solar active regions and in flares' energy release, we show that the model is able to reproduce the bulk of the above statistical properties. We thus underline two main conclusions: (i) A global, statistical approach for the study of rapid energy dissipation and magnetic field line annihilation in complex, magnetized plasmas may be of equal importance with the localized, small-scale Magnetohydrodynamic (MHD) simulations, and (ii) refined SOC models are needed to establish a more physical connection between the cellular automata evolution rules and the observations.
\end{abstract}

Key words. Sun: activity - Sun: corona - Sun: flares - Sun: magnetic fields - Sun: X-rays

\section{Introduction}

After several decades of observations of the flaring solar corona, it is now well recognized that the solar corona is a highly non-linear dynamical system, seat of many phenomena related to magnetic energy releases in a large range of sizes which occur on time scales ranging from a few seconds or less, to hours. Numerous observations on flaring activity (e.g. Datlowe et al. 1974; Lin et al. 1984; Dennis 1985; Crosby et al. 1993; Lee et al. 1993; Pearce et al. 1993; Biesecker 1994; Bromund et al. 1995; Crosby et al. 1998b) have shown that the frequency distribution functions of the most representative flare parameters are well-defined, robust power laws extending over a number of decades. This was conceived as the demonstration that

Send offprint requests to: M. K. Georgoulis, e-mail: georgoul@astro.auth.gr the rapid dynamical evolution in the flaring corona is governed by self-similar, scale-invariant statistics.

The results of the most recent statistical studies performed on hard X-ray bursts have been summarized in Crosby et al. (1998b). Most of the distributions are well represented by power laws extending over several decades. The small deviations in the values of the logarithmic slopes derived from different hard X-ray data sets (e.g. Crosby et al. 1993; Bromund et al. 1995) are so far attributed to differences in instrument sensitivities or different regression methods. It has also been reported that, although the flare occurrence rate varies by more than one order of magnitude between the solar maximum and minimum, the distribution functions of typical parameters of flares are insensitive to the phases of the 11-year solar cycle (Crosby et al. 1993; Biesecker 1994; Bromund et al. 1995). Despite 
this long-term independence of the statistical properties of flaring activity with the phase of the solar cycle, however, it has been shown that the slopes of the distribution functions may depend on the phase of the 154-day periodicity in the occurrence rates of flares (Bai 1993; Bromund et al. 1995). This periodicity, first discovered by Rieger et al. (1984) and later formulated by Sturrock \& Bai (1992) and Bai \& Sturrock (1993), is thought to be the most prominent harmonic of a fundamental period of 25.5 days. It is thus suggested that the characteristics of the individual component distribution functions are lost when the entire data set is taken into account. This result may indicate that the featureless, simple self-similar picture of the observed distribution functions is the final product of the convolution of significantly different component distribution functions. Therefore, it appears likely that a number of power-law distribution functions with different scaling indices may merge to provide the long-term, overall distribution functions of flaring events.

A statistical study of solar flares was also performed on the burst observations in the deka-keV X-ray range provided by the Danish Wide Angle Telescope for Cosmic Hard X-rays (WATCH) aboard the Russian GRANAT spacecraft (Crosby et al. 1998a,b). The WATCH solar burst catalogue consists of solar X-ray bursts observed in the energy range above $10 \mathrm{keV}$ during the maximum of solar cycle 21 (January 1990-July 1992). The WATCH solar burst catalogue is available on-line at the Centre de Données (CDS) astronomiques de Strasbourg and more information about the WATCH instrument and the data selection process can be found in Crosby (1996) and Crosby et al. (1998a). The aim of the statistical study was: (i) to determine whether the global characteristics of the distribution functions, as well as correlations between typical parameters of events observed in the hard X-ray domain hold also for the deka-keV range (an energy channel which has not been extensively studied in the past), (ii) to investigate whether the overall frequency distributions of flares are the convolution of different distribution functions when built on parts of the overall data set, and (iii) to investigate correlations between the size of events and the elapsed time between them, either for the entire dataset, or for events associated with the same active region. The results of this study are summarized in Sect. 2.

Any successful model for solar flares should be able to explain (or at least to reproduce) the results of statistical studies of solar flares. The actual physical processes leading to a flare are traditionally the aim of MHD and MHD Turbulence theoretical models. Admittedly, though, those "strict" physical models seem incapable to address the question of statistics, due to computational limitations. For this reason, a new class of models has been developed lately to tackle the problem. The concept of Self-Organized Criticality, first introduced by Bak et al. (1987, 1988) and further formulated by Kadanoff et al. (1989) has provided the background of these phenomenological models. SOC encloses a number of features that seem applicable in the case of active regions. It deals with nonlinear, externally driven dynamical systems. Their inherently nonlinear behavior is directed in the course of time towards a self-organized critical state with respect to some pre-defined critical threshold. Once being in this state, the statistical behavior of the system is described by extended self-similarity and scaling laws, as a result of the internal self-organization. As already mentioned, the interpretation SOC attempts to attribute is rather phenomenological. The aim of the concept is to capture the global behavior of intrinsically self-organized critical systems and not to analyze per se the actual physical processes that lead to self-organization.

The first application in the case of active regions and solar flares was attempted by Lu \& Hamilton (1991). They assumed that active regions may be viewed as selforganized critical systems, externally driven by smallscale photospheric shuffling of the footpoints of coronal loops. Scaling laws and self-similarity in the distribution functions were thereafter attributed to this internal selforganization. Their cellular automaton SOC model could materialize a situation that included cascades of elementary events, fragmented energy release and other aspects, commonly appearing in fully turbulent systems, as solar active regions are thought to be.

A comparison of the model's results with observations was attempted by Lu et al. (1993), using the database constructed by ISEE 3/ICE satellite observations. The model was able to reproduce reasonably well most of the statistical properties of flares. Specifically (i) the distribution functions of event parameters were robust scaling laws with slopes close to the ones obtained by data, (ii) correlations between typical event parameters were predicted, and (iii) the insensitivity of the elapsed time between two successive events and the event size was shown. A weak point in the model of $\mathrm{Lu} \&$ Hamilton (1991) and Lu et al. (1993), however, was that the model could not predict any significant variability of the scaling indices in the distribution functions of the simulated events.

A refined SOC model for solar flares was introduced by Vlahos et al. (1995) and Georgoulis et al. (1995). In addition to previous predictions, this model could predict the existence of discrete, low-energy populations of events in the distribution functions, governed by steeper scaling laws. Such a result was in favor of the "nanoflaring" scenario (Parker 1988, 1989) and, moreover, it was compatible with the theoretical assertion of Hudson (1991) about the statistical properties of the hypothetical nanoflares. In a further refinement, Georgoulis \& Vlahos $(1996,1998)$ tackled the problem of the variability of scaling indices in the distribution functions, introducing a highly variable, non-linear external driver. The physical justification of such a driver is attributed both to photospheric shuffling and the emergence of new magnetic flux from the convection zone in active regions. A highly variable driver gives rise to variable slopes in the distribution functions of the events obtained (for a parametric study, see Georgoulis \& Vlahos 1998). 
In the present work, we attempt a systematic comparison between the results of the "Statistical Flare Model" (Georgoulis \& Vlahos 1996, 1998) and the statistical properties of WATCH flare data (Crosby 1996; Crosby et al. 1998a,b). In addition to similar comparisons performed by Lu et al. (1993), we attempt to address the issue of different scaling indices for different sub-sets of the model results, assuming analogous distinguishing criteria as those adopted in Crosby (1996) and Crosby et al. (1998b). To our knowledge, this study is the most tentative "feasibility test" of a SOC model, in view of complex observational results. We need to emphasize, however, that a one-toone comparison between the model results and WATCH flares is subject to a number of caveats: (i) A physical link between model units and data units is lacking. The model units are arbitrary, while the data is expressed in detector-dependent quantities (counts and counts $\mathrm{s}^{-1}$ ). The conversion between detector units, photon fluxes and released energies has not been done since, for most of the recorded bursts, no photon spectral information is available. (ii) The energy range of WATCH observations may contain contribution from thermal plasma around a few $\mathrm{keV}$. However, various characteristics of the bursts observed by WATCH above $10 \mathrm{keV}$ still suggest that the radiation above $10 \mathrm{keV}$ is a good indicator of the primary energy release in the flare and not of the thermal response of the medium (see Crosby 1996; Crosby et al. 1998b for more details and examples of observations). (iii) A number of physical processes which certainly modify the energy release process and the temporal profiles of flares are by no means included in the simulation. Such processes are, for example, particle acceleration, particle transport and energy losses in the flaring medium (for a review, see Trottet \& Vilmer 1997). Therefore, although the comparison between the model and the data may also include rise and decay times of events, we do not perform such a comparison in the present study.

The paper is structured as follows: in Sect. 2 we summarize the statistics of flares observed by WATCH. In Sect. 3 we briefly outline the main aspects of the Statistical Flare Model. In Sect. 4 a detailed comparison between the model results and WATCH data is performed, while in Sect. 5 we summarize our findings and we discuss them within the general framework of the dynamical evolution in solar active regions.

\section{Statistical properties of the deka-keV X-ray flares observed by WATCH}

The statistical study performed on the solar flare observations in the deka-keV range obtained with the WATCH experiment on board the GRANAT satellite is described in detail in Crosby et al. (1998b). Although the energy range around $10 \mathrm{keV}$ may contain contribution from the thermal plasma around a few $\mathrm{keV}$, a number of characteristics of the events observed by WATCH above $10 \mathrm{keV}$ suggests that non thermal emission can be observed as low as $10 \mathrm{keV}$ and that this energy range may provide information on the primary energy release and not necessarily on the thermal response of the plasma: (i) The comparison of time profiles in the $10-30 \mathrm{keV}$ range with respect to time profiles of the same event in the soft $\mathrm{X}$-ray domain observed by GOES, and/or with the ones at typical hard X-ray energies shows indeed that nonthermal emission can be observed as low as $10 \mathrm{keV}$. (ii) The Neupert effect generalized by the relationship between the time derivative of the soft X-ray time profile observed by GOES with the hard X-ray light curve is also observed for some events between the WATCH time profiles and the GOES ones around a few keV (see Crosby et al. 1998b for more details). The results of the statistical study are summarized here with some emphasis on the characteristics of the distributions and on the correlations between parameters that will be tentatively reproduced by the Statistical Flare Model of Georgoulis \& Vlahos $(1996,1998)$. As in previous observational studies at higher X-ray energies, it is found that the distribution function of the peak count rate of 1518 hard X-ray flares detected by WATCH is a well-defined, extended power law, for almost three orders of magnitude, with a slope $-1.59 \pm 0.05$ (Fig. 1b; see also Crosby et al. 1998b). The distribution of total counts of flares is also a well-defined power law, extending over three orders of magnitude, with an index of $-1.39 \pm 0.02$ (Fig. 1a). The total-duration distribution function cannot be represented by a single power law (Fig. 1c). Instead, it can be fitted either by two power laws with indices $-1.15 \pm 0.04$ (for durations $\leq 10^{3} \mathrm{~s}$ ) and $-2.25 \pm 0.08$ (for durations $>10^{3} \mathrm{~s}$ ), or, as suggested by $\mathrm{Lu}$ et al. (1993) by a power law with index $-1.09 \pm 0.03$ followed by an exponential roll-over beyond a cut-off value $D_{\max }=2100 \pm 100 \mathrm{~s}$. Even in this case, the power law predicted by Lu et al. (1993) is much steeper compared to the one derived from WATCH data. The difference between the total-duration distribution function presented here and the ones observed at higher energies may be due to the fact that several X-ray components exist in the energy range around $10 \mathrm{keV}$. However, it is worthwhile noting that the power law slope for large durations is relatively consistent with what is found at higher energies for similar durations (see Crosby et al. 1993). The two slopes found here in the total-duration distribution function may thus be simply due to systematic effects resulting from the definition of the duration. The latter is defined as the time interval when the count rate is above background. This may induce first a reduced sensitivity for shorter duration bursts (flatter slope and cut-off at low values) and second some strong influence on this parameter of the thermal contribution. The uncertainty in start and end time of a burst may also explain larger deviations from simple power laws of this distribution function.

Additionally, it was investigated whether the overall distribution functions of the peak count rate and the total counts of flares are built of, or can be decomposed into, a number of independent distributions.Thus, dividing the entire data base in sub-groups of events with different duration intervals (the intervals being chosen so as to 

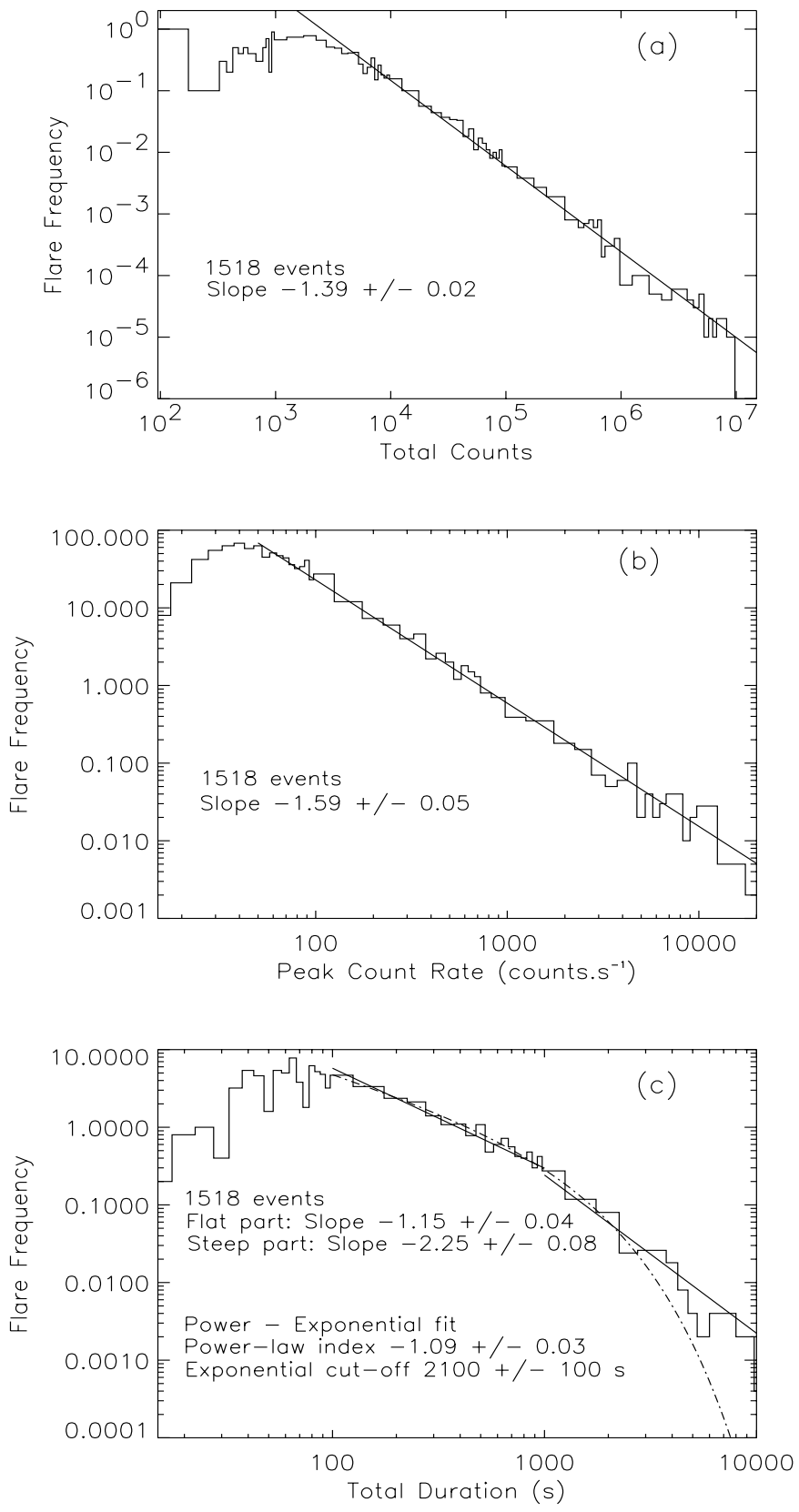

Fig. 1. a-c) Distribution functions of the parameters of the X-ray flares observed by WATCH. Distributions refer to a) total counts in the flare, $\mathbf{b}$ ) peak count rate and $\mathbf{c}$ ) total flare duration

achieve sufficient statistics in each sub-group), it is found that the frequency distributions of the total counts (peak count rates) are still well represented by robust power-law distributions, with a slope systematically decreasing with increasing duration. For short duration events $(<200 \mathrm{~s})$ the slope of the total-counts (peak-count-rate) distribution function is $-2.38 \pm 0.07(-2.17 \pm 0.07)$ while for the longer duration events $(>1000 \mathrm{~s})$ it is $-1.28 \pm 0.06$ $(-1.15 \pm 0.05)$. This effect is clearly seen in Fig. 2 which shows the frequency distributions of the total counts for both the shortest- and longest-duration events. The systematic variability of the scaling indices for different

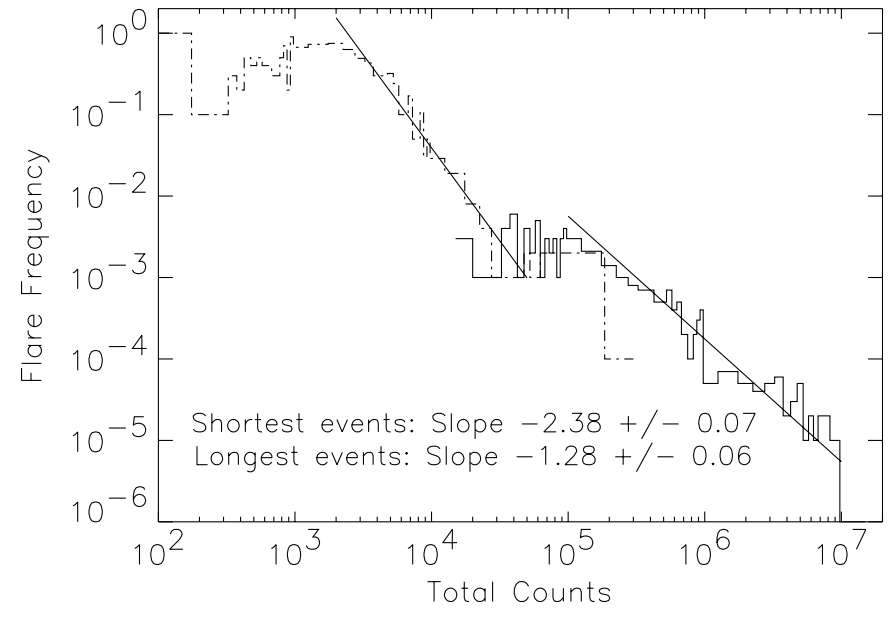

Fig. 2. The distribution functions of total counts for the two extreme total-duration sub-groups in the WATCH data set

Table 1. Power-law indices $\delta$ of the distribution functions of total count rates for different sub-groups of WATCH events defined by their total duration. The notation used is $N_{\text {fit }}$ : number of events used for the best fit. $N_{\text {tot }}$ : total number of events included in the distribution

\begin{tabular}{llll}
\hline \hline $\begin{array}{l}\text { duration intervals } \\
\text { (seconds) }\end{array}$ & slope & $N_{\text {fit }}$ & $N_{\text {tot }}$ \\
\hline$D>6.5$ & $-1.39 \pm 0.02$ & 1232 & 1518 \\
$6.5<D<200$ & $-2.38 \pm 0.07$ & 304 & 647 \\
$200<D<600$ & $-1.87 \pm 0.05$ & 309 & 473 \\
$600<D<1000$ & $-1.57 \pm 0.06$ & 131 & 186 \\
$D>1000$ & $-1.28 \pm 0.06$ & 164 & 212 \\
\hline \hline
\end{tabular}

duration intervals (see Table 1 for total-counts distributions) shows that the overall power-law frequency distribution of the total counts of the observed events is built of a number of component distribution functions which are power laws, as well. Table 1 further shows that the longest and, as a mean, the largest events are found to control the overall distribution although they are less numerous than the shortest and, as a mean, the weakest events. Totalduration frequency distributions are also built for two subgroups of events defined by the range of peak count rates and it is found that when reproduced by two power laws, both power laws are significantly flatter for the subgroup with the largest peak count rates. If represented by a power law with an exponential roll-over, the power law is flatter for the subgroup with the largest peak count rates, while the value of the exponential cut-off $D_{\max }$ increases. A similar behavior is seen in the frequency distributions of rise and decay times (Crosby et al. 1998b).

The results concerning correlation scatter plots of total duration, peak count rate, total counts, rise and decay times are summarized in Tables 2 and 3. It is interesting to note that, despite the considerable dispersion of data points, a power-law relationship can still be found between the parameters (Table 2; see also Fig. 12 in Crosby et al. 1998b) and moderate-to-high correlation coefficients are evaluated (Table 3 ). The above-mentioned correlation 
Table 2. Scaling indices of the correlation scatter plots between different parameters of the observed X-ray flares. The notation used is $T$ : total counts. $P$ : peak count-rate. $D$ : total duration. $R T$ : rise time. $D T$ : decay time

\begin{tabular}{|c|c|c|c|c|c|}
\hline & $\begin{array}{l}T \\
\text { (counts) }\end{array}$ & $\begin{array}{l}P \\
\left(\text { counts s }^{-1}\right)\end{array}$ & $\begin{array}{l}D \\
(\mathrm{~s})\end{array}$ & $\begin{array}{l}R T \\
(\mathrm{~s})\end{array}$ & $\begin{array}{l}D T \\
(\mathrm{~s})\end{array}$ \\
\hline$T$ & 1.0 & $0.60 \pm 0.01$ & $0.53 \pm 0.02$ & $0.47 \pm 0.02$ & $0.53 \pm 0.01$ \\
\hline$P$ & $1.31 \pm 0.02$ & 1.0 & $0.54 \pm 0.03$ & $0.49 \pm 0.02$ & $0.54 \pm 0.03$ \\
\hline$D$ & $1.44 \pm 0.02$ & $0.68 \pm 0.04$ & 1.0 & $0.94 \pm 0.01$ & $1.02 \pm 0.01$ \\
\hline$R T$ & $1.17 \pm 0.03$ & $0.50 \pm 0.03$ & $0.83 \pm 0.01$ & 1.0 & $0.75 \pm 0.02$ \\
\hline$D T$ & $1.38 \pm 0.02$ & $0.66 \pm 0.03$ & $0.93 \pm 0.01$ & $0.75 \pm 0.02$ & 1.0 \\
\hline
\end{tabular}

coefficients are the standard linear correlation coefficients linking the logarithms of the studied parameters. The correlations found here are consistent with what was previously observed at higher energies with HXRBS/SMM (Crosby et al. 1993). It is finally found that there is no correlation between the elapsed time interval between successive flares from the same active region and the peak intensity of a flare (see Crosby 1996; Crosby et al. 1998b for details).

\section{The Statistical Flare Model}

The aim of this section is to summarize the general aspects of the cellular automaton SOC Statistical Flare Model, used in this paper to reproduce the statistical properties of flaring activity and described in Georgoulis \& Vlahos $(1996,1998)$. The model assumes that the solar corona is a driven dissipative dynamical system, which self-organizes gradually and locally (in active regions) to a state of marginal stability with respect to a pre-defined critical threshold. In case the local values of some degree of freedom in the system exceed this threshold, the system experiences avalanches of events with durations and sizes governed by scale-invariant, self-similar properties. We shall simply recall here only the important aspects of the model used in the next section. Let us also recall the output parameters of the model: a) the total duration $D$ of an event is defined as the total number of time-steps from the onset to the complete relaxation of the event, b) the total energy $T$ of an event is the accumulated released energy during the event's duration and c) the peak luminosity $P$ of an event is the maximum energy registered in a time step during the event (both $T$ and $P$ are expressed in arbitrary model units).

In most versions of SOC models (Lu \& Hamilton 1991; Lu et al. 1993; Vlahos et al. 1995), the driver used to represent the perturbation applied to a given point in a magnetic configuration was roughly constant and small compared to the critical threshold characterizing the SOC state. Although a case with a variable driver has been examined by Galsgaard (1996), the magnitude of the driver still does not reach the magnitude of the critical threshold introduced. This small-scale driving of the system is giving rise to a roughly unique value in the scaling indices of the frequency distributions of typical event parameters. However, as discussed in more detail by Georgoulis \& Vlahos (1998), in the actual system of the solar corona,
Table 3. Linear correlation coefficients in the scatter plots between different parameters of the observed X-ray flares. The notation used is $T$ : total counts. $P$ : peak count-rate. $D$ : total duration. $R T$ : rise time. $D T$ : decay time

\begin{tabular}{llllll}
\hline \hline & $T$ & $P$ & $D$ & $R T$ & $D T$ \\
$($ counts $)$ & $\left(\right.$ counts s $\left.^{-1}\right)$ & $\begin{array}{l}\text { (s) } \\
(\mathrm{s})\end{array}$ & $(\mathrm{s})$ \\
\hline$T$ & 1.0 & 0.89 & 0.87 & 0.76 & 0.86 \\
$P$ & 0.89 & 1.0 & 0.61 & 0.50 & 0.62 \\
$R T$ & 0.76 & 0.50 & 0.88 & 1.0 & 0.74 \\
$D T$ & 0.86 & 0.62 & 0.96 & 0.74 & 1.0 \\
\hline \hline
\end{tabular}

the driver which triggers energetic phenomena in active regions can be either small-amplitude shuffling due to the random motions of the loops' photospheric footpoints, or large-amplitude modification of the topology because of the emergence of new magnetic flux from the convection zone (Alfvénic perturbations within an existing coronal loop or the emergence of new flux tubes). Given the generally accepted turbulent nature of the convection zone and the photosphere, Georgoulis \& Vlahos $(1996,1998)$ introduced a driver of the form $P(\delta B)=A(\delta B)^{-\alpha}$ where $P(\delta B)$ is the probability of a certain increment $\delta B$ (modeling either the emergence of new flux or the local change in flux due to shuffling) to occur and $\alpha$ is the driver's scaling exponent. Such a driver allows to take into account scenarios of energy storage and subsequent release, as well as instantaneous relaxation of excessively unstable configurations. Georgoulis \& Vlahos (1998) then found that scaling laws are maintained in the frequency distributions and in the correlations of the event parameters and, moreover, that the variability of the scaling index $\alpha$ induces a respective variability in the resulting power-law indices. In addition, the relation between the driver's exponent $\alpha$ and the resulting power-law exponents is well defined. It thus appears that a modified SOC state survives even with a highly variable, non-linear driver, provided that its statistical mean is in all cases well below the critical threshold. In the next section, the properties of the variable driver and the consequent variability of the output indices will be used to map the scaling index of the WATCH peak-countrate distribution function as compared to the simulated peak-luminosity frequency distribution. 


\section{Model results versus WATCH observations}

The purpose of this section is to provide a comparison of the results of the simulation with similar statistical results obtained from the WATCH data base of solar X-ray flares.

We emphasized in the Introduction that a direct comparison between the results of the simulation and the statistical properties deduced from observations must be considered with some care. The comparison is intended here only to show that SOC models are able to reproduce most of the tendencies seen in the statistical properties of solar flares. The limitations preventing to achieve a direct comparison are numerous and many important elements, both in the model and in the observations, are missing:

- the arbitrary units of time and energy used in the simulation should be related to physical units in the solar corona. Such a relation is lacking;

- the conversion of the released energy in either thermal or non-thermal distributions of particles in the solar plasma as well as the radiation from these particles is not considered in the simulation;

- the present observational statistical study is based on detector-dependent quantities as physical quantities cannot be obtained for the whole database, because of the lack of available photon spectral information for most bursts. Furthermore, the energy range of WATCH observations may contain contribution from thermal plasma around a few keV. However, as discussed in the previous sections, some characteristics of the bursts above $10 \mathrm{keV}$ still suggest that the radiation above $10 \mathrm{keV}$ is a good indicator of the primary energy release in the flare and not only of the thermal response of the medium.

\subsection{Frequency distributions of event parameters}

In Fig. 3 we present the distribution functions of the total energy, peak luminosity and total duration of events derived from the Statistical Flare Model. Following the parametric study performed in Georgoulis \& Vlahos (1998), the scaling exponent $\alpha$ of the driver has been selected in such a way as to map the scaling index of the peak count-rate of WATCH data with the scaling index of the simulated peak-luminosity distribution function. This is achieved for $\alpha=1.1$.

It is clear that, in all distribution functions obtained from the model (Fig. 3), there is a double scaling behavior which is not observed, either in the WATCH distributions, or in any other distributions deduced from X-ray observations. The observational distribution functions are therefore compared in the following to the flat (hard) parts of the distribution functions obtained from the simulation. The flare observations probably correspond to the simulated distribution of intense events leading to the harder distributions, while the simulated softer distributions (tentatively associated with nanoflares) do not correspond yet to any observed distributions of parameters of X-ray flares.
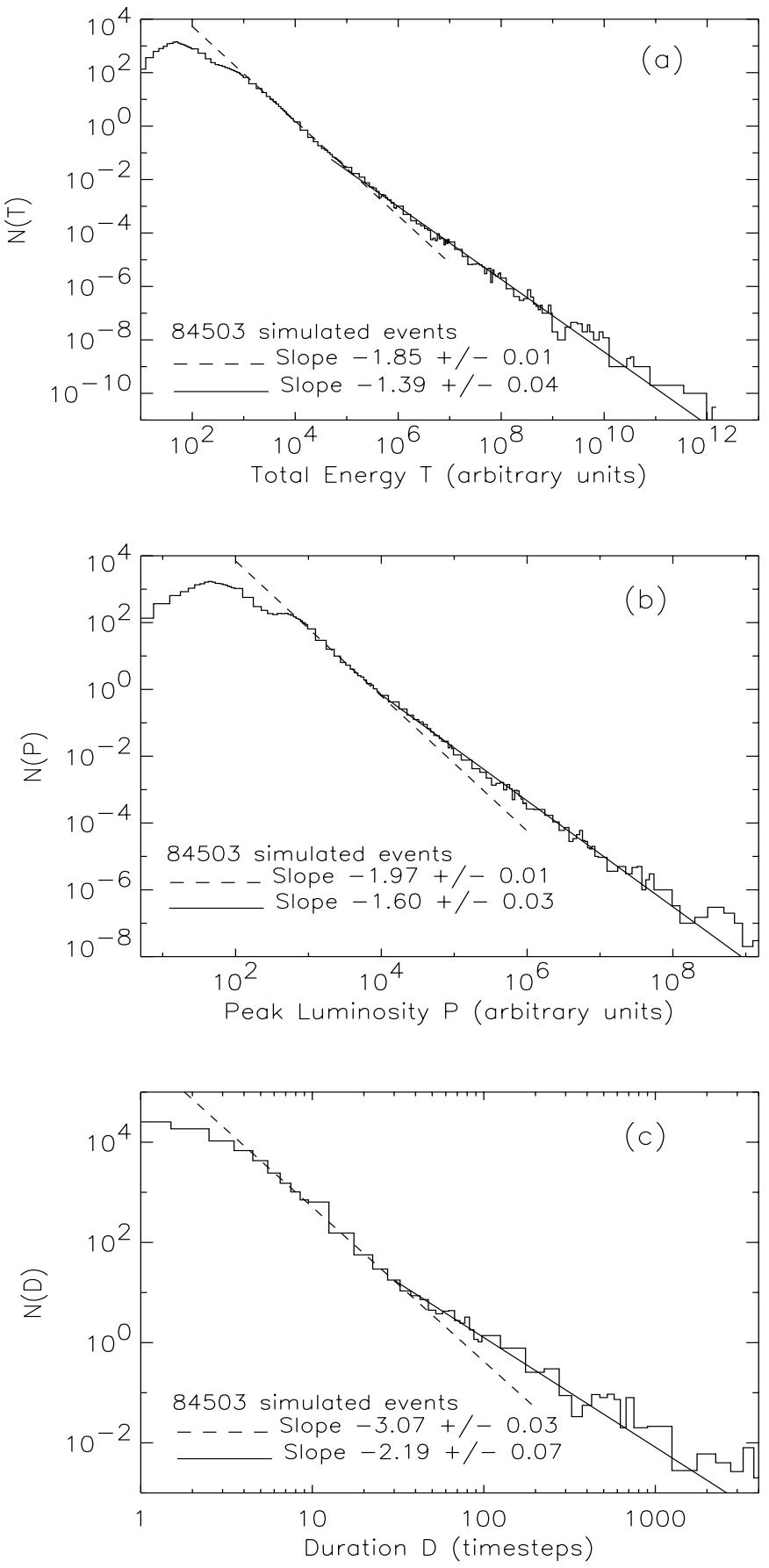

Fig. 3. a-c) Distribution functions of the parameters of events simulated by the Statistical Flare Model. Distributions refer to a) total energy, b) peak luminosity and c) duration of events

From the distribution functions of Fig. 3 it is evident that the attempt to fix $\alpha$ in order to reproduce the observational peak-count-rate distribution function leads to a consistency between the scaling indices of (i) the simulated total-energy and the observed total-counts distribution functions and (ii) the simulated total-duration distribution function and the steep part of the observed total-duration one. The model provides a (flat) index of -1.60 for the peak-luminosity distribution (Fig. 3b) to be compared with the index -1.59 for the observational 
peak-count-rate distribution (Fig. 1b), while the totalenergy distribution index of -1.39 (Fig. 3a) is to be compared with its observational counterpart of -1.38 for the total-counts distribution of WATCH flares (Fig. 1a). Some discrepancy appears, however, when one compares the distribution functions of total duration for the model and the observations (Figs. 3c and 1c respectively). The duration distribution function of WATCH flares cannot be fitted by a single power law but, instead, it appears to enclose a flatter power law for short durations (100 s $<D<1000 \mathrm{~s})$, while for larger durations there is a steeper power law with an index -2.21 , closely related to the model's flat power-law index of -2.19 . The double power law shown in WATCH data is thus not reproduced. A bias in the observations cannot, however, be completely ruled out since the flat part of the observed total-duration distribution shown in Fig. 1c has not been observed by other experiments. Among the different observational parameters, the event duration is indeed the one for which uncertainties may be the most important (due to the definition) and also the parameter which is the most dependent on physical processes not considered in the statistical flare model. This bias may also account for the discrepancy found when one compares the observed and the simulated total-duration distribution functions (see above). An important point to notice however is that a good agreement is found between the simulations and the observations for the longest events for which the uncertainty in determing the parameters is less important.

The total number of discrete events included in the distribution functions of Fig. 3 is 84 503. An examination of those events' temporal profiles revealed a fraction of them, equal to 12888 , which enclose fine temporal structures. In the present context, the term "fine temporal structure" means that discrete ( $\geq 1$ time-step) rise and decay times can be defined. Events with durations equal to 1 or 2 timesteps, as well as events with their peak located at their first time-step, are thus excluded from the above selection. Given the large number of events with discrete temporal profiles which guarantees sufficient statistics, one may wish to extend the above analysis pursuing also a comparison between the rise and decay times of the observed and the simulated events. Such an analysis, however, is un-warranted, since this task probably lies beyond the capabilities of the Statistical Flare Model. Indeed, rise and decay times are very dependent on a number of physical processes (particle transport, trapping and energy losses in the flaring medium) which are not considered at all in this simulation.

\subsection{Division of events in sub-groups}

Following the decomposition of WATCH data in subgroups, we investigate whether the observed variability of scaling indices of the distribution functions for subgroups of events filtered either by their total duration, peak count rates or total energy can also be reproduced
Table 4. Power-law indices $\delta$ of the distribution functions of total energy for different total duration sub-groups of the simulated events. The entire total-energy distribution function of Fig. 3a is included. The notation used is $N_{\text {fit }}$ : number of events used for the best fit. $N_{\text {tot }}$ : total number of events included in the distribution

\begin{tabular}{llll}
\hline \hline $\begin{array}{l}\text { duration intervals } \\
\text { (timesteps) }\end{array}$ & slope & $N_{\text {fit }}$ & $N_{\text {tot }}$ \\
\hline $1<D<7$ & $-2.75 \pm 0.07$ & 3574 & 77455 \\
$7<D<35$ & $-1.51 \pm 0.04$ & 3343 & 6368 \\
$35<D<100$ & $-1.39 \pm 0.02$ & 391 & 481 \\
$D>100$ & $-1.26 \pm 0.08$ & 181 & 199 \\
\hline \hline
\end{tabular}

by the Statistical Flare Model. First, the total duration of the simulated events is considered as the filter of division. The simulated events are divided in different sub-groups defined by four duration intervals. The limits of the different duration intervals are chosen as to provide sufficient statistics in each interval. The total-energy distribution function for each sub-group is then constructed taking into account both the entire data set of the simulation (that is both scaling laws of the total-energy frequency distribution of Fig. 3a) or the events belonging only to the hard (flatter) part of the total-energy distribution function. These latter simulated events should be more representative of the observations, which do not include the soft part of the distribution. The results are summarized in Table 4 (for the entire total-energy distribution function) and Table 5 (for the events belonging to the hard part of the total-energy frequency distribution). It should be noted at this point that the filtering causes the bimodal power-law behavior seen in Fig. 3 to cease. The component distribution functions, either for the entire population of events or for its hard part alone, are described by single power laws. Figure 4 depicts the total-energy distribution functions of events belonging to the two extreme duration sub-groups. As it can be seen from Tables 4 and 5, as well as from Fig. 4, the variability of scaling indices for the distribution functions of events filtered by their duration is also an intrinsic property of the Statistical Flare Model. As in the observations (cf. Fig. 2 and Table 1), there is a clear tendency of obtaining significantly different powerlaw indices for different sub-groups of events. The effect is, however, more pronounced in the simulation. This may be due to the fact that the different sub-groups (in the observations and the simulation) have been chosen according to their specific scales of durations and the two kinds of units are not yet linked physically. In any case, it is clear that introducing a filter to focus on shorter events, reveals increasingly steeper distributions than the ones obtained for the bulk of events. Remarkably, these component distributions maintain the power-law shape. Otherwise put, a well-defined, observed or simulated, power-law distribution function could be viewed as a superposition of strikingly different power-law distribution functions.

The variability of the scaling index for different sub-groups of events filtered with respect to different 

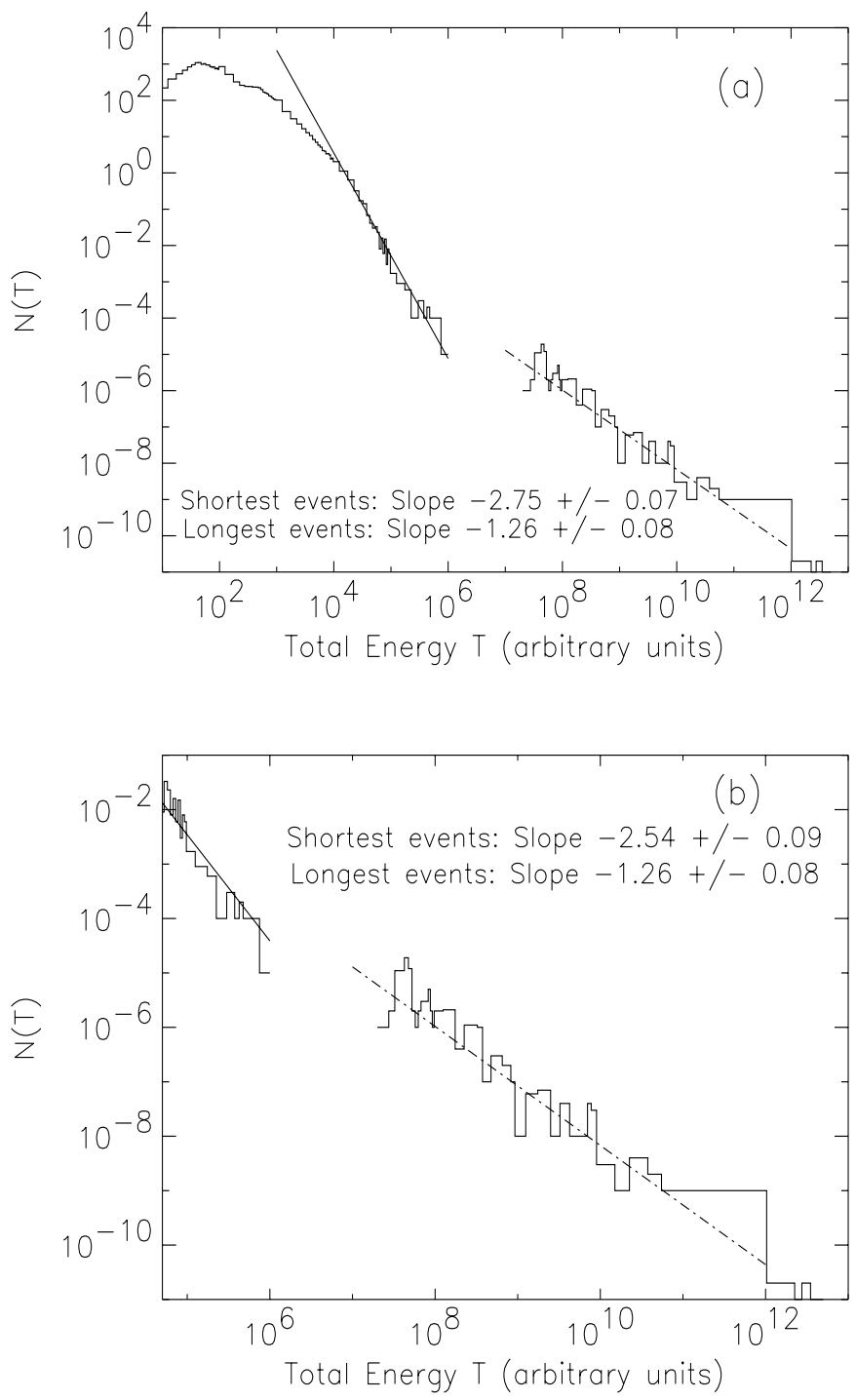

Fig. 4. a and b) The distribution functions of total energy for the two extreme total-duration sub-groups of the simulated events. Distributions refer to a) the entire total-energy distribution function of Fig. 3a and $\mathbf{b}$ ) the hard part of Fig. 3a

total-energy intervals has also been investigated in the results of the simulation. The total-duration distribution functions for each sub-group are constructed and the scaling indices are evaluated. Again, the double power-law behavior seen in the distribution function of Fig. 3c breaks down. The results, obtained for three total energy intervals, are summarized in Table 6 for events with $T>110^{3}$ arbitrary units (i.e. for events above the low total-energy cut-off in the distribution functions of Fig. 3). Moreover, the effect of obtaining steeper power laws when larger events are excluded is clearly seen in Table 6 . It is worth reporting that a similar, although less clear, effect can be found for sub-groups based on different peak count-rates intervals in the observations (see, e.g. Crosby 1996).
Table 5. Power-law indices $\delta$ of the distribution functions of total energy for different total duration sub-groups of the simulated events. Only the hard part of the total-energy distribution function of Fig. 3a is included. The notation used is $N_{\text {fit }}$ : number of events used for the best fit. $N_{\text {tot }}$ : total number of events included in the distribution

\begin{tabular}{llll}
\hline \hline $\begin{array}{l}\text { duration intervals } \\
\text { (timesteps) }\end{array}$ & slope & $N_{\text {fit }}$ & $N_{\text {tot }}$ \\
\hline$D>1$ & $-1.39 \pm 0.02$ & 1459 & 1567 \\
$1<D<7$ & $-2.54 \pm 0.09$ & 168 & 168 \\
$7<D<35$ & $-1.60 \pm 0.07$ & 719 & 719 \\
$35<D<100$ & $-1.39 \pm 0.02$ & 391 & 481 \\
$D>100$ & $-1.26 \pm 0.08$ & 181 & 199 \\
\hline \hline
\end{tabular}

Table 6. Power-law indices $\delta$ of the total-duration distribution function for different total-energy intervals of the simulated events. The entire duration distribution function of Fig. $3 \mathrm{c}$ is included. The notation used is $N_{\text {fit }}$ : number of events used for the best fit. $N_{\text {tot }}$ : total number of events included in the distribution

\begin{tabular}{llll}
\hline \hline $\begin{array}{l}\text { total-energy intervals } \\
\text { (arbitrary units) }\end{array}$ & slope & $N_{\text {fit }}$ & $N_{\text {tot }}$ \\
\hline $110^{3}<T<110^{6}$ & $-2.83 \pm 0.04$ & 11826 & 31629 \\
$110^{6}<T<110^{9}$ & $-2.10 \pm 0.11$ & 520 & 762 \\
$T>110^{9}$ & $-1.43 \pm 0.12$ & 47 & 47 \\
\hline \hline
\end{tabular}

\subsection{Correlation scatter plots}

In this section, we investigate correlations between the different parameters of the simulated events and compare the results with the respective correlations between observed flare parameters. As already mentioned, in the absence of a well-established physical link between the arbitrary model units and the data units, we have performed a crude association between the total energy of the simulated events and the observed total counts of flares, the peak luminosity and the observed peak count rate and finally the total duration (in time-steps) and the observed total duration (in seconds). As shown in Georgoulis \& Vlahos (1998), the scatter plots obtained for the parameters of the model show a double scaling behavior, as in the frequency distributions of the parameters. The low-energy, soft populations of events provide steeper scaling laws in the scatter plots. For the purpose of comparison with data and in consistency with the comparisons between scaling indices of the distribution functions, we consider in the following only the hard (flat) correlation scaling laws. This selection reveals a number of 1567 simulated events. The indices of the correlation scaling laws and the (linear) correlation coefficients obtained from the model are summarized in Tables 7 and 8, respectively. Comparing quantitatively Tables 2 and 7, it can be seen that there is a fair agreement with observations for correlations between peak activity $P$ (peak count rate and peak luminosity) vs. total activity $T$ (total counts and total energy - indices 0.60 and 
Table 7. Scaling indices of the correlation scatter plots between different parameters of the simulated events. The notation used is $T$ : total counts. $P$ : peak count-rate. $D$ : total duration

\begin{tabular}{llll}
\hline \hline & $T$ & $P$ & $D$ \\
& (arbitrary units) & (arbitrary units) & \begin{tabular}{l} 
(timesteps) \\
\hline$T$
\end{tabular} \\
$P$ & 1.0 & $0.64 \pm 0.01$ & $0.36 \pm 0.01$ \\
$D$ & $2.70 \pm 0.01$ & 1.0 & $0.55 \pm 0.01$ \\
\hline \hline
\end{tabular}

Table 8. Linear correlation coefficients in the scatter plots between different parameters of the simulated events. The notation used is $T$ : total counts. $P$ : peak count-rate. $D$ : total duration

\begin{tabular}{llll}
\hline \hline & $T$ & $P$ & $D$ \\
& (arbitrary units) & (arbitrary units) & \begin{tabular}{l} 
(timesteps) \\
\hline$T$
\end{tabular} 1.0 \\
$P$ & 0.98 & 0.98 & 0.75 \\
\hline \hline
\end{tabular}

0.64 from the data and the model, respectively) and total duration $D$ vs. peak activity $P$ (indices 0.54 and 0.55 from the data and the model, respectively). The larger discrepancy is found for the scatter plot of total duration $D$ vs. total activity $T$, where the scaling index $0.36 \pm 0.01$ obtained by the model is to be compared with the index $0.53 \pm 0.02$ from WATCH data. It must be noted that these parameters are the ones for which the temporal profiles of events may be determinative both in the observations where several X-ray components may be mixed and in the simulations where losses are not taken into account. To our opinion, this is another indication that the comparison between the model and observations concerning the parameters describing in more detail the temporal behavior of events is un-warranted, for the reasons discussed in Sect. 4.1.

The comparison of the correlation scatter plots of $T$ vs. $P, T$ vs. $D$ and $P$ vs. $D$ (the inverse of $P$ vs. $T, D$ vs. $T$ and $D$ vs. $P$, respectively), between WATCH data and model results, leads to a somewhat surprising result. In the scatter plots of the simulated events, where the correlation coefficients (Table 8) are, in general, larger compared to the correlation coefficients calculated from the observations (Table 3 ), both the rule $X \propto Y^{\delta_{\mathrm{c}}}$ and its inverse, $Y \propto X^{1 / \delta_{\mathrm{c}}}$ are approximately valid $(X, Y \equiv\{T, P, D\}$ and $\delta_{\mathrm{c}}$ is the scaling index). This is not the case, however, in the WATCH data set where a considerable dispersion of the data points around the theoretical power law violates the above mathematically equivalent rules. For this reason, the scaling indices of $T$ vs. $P, T$ vs. $D$ and $P$ vs. $D$ are different between the model results and the observations (1.54 and $1.31,2.70$ and $1.44,1.75$ and 0.68 , respectively). A relative agreement appears between the scaling indices of $T$ vs. $P$. Not surprisingly, the correlation coefficients for the observations and the model results in this case are the highest obtained (0.89 and 0.98, respectively).
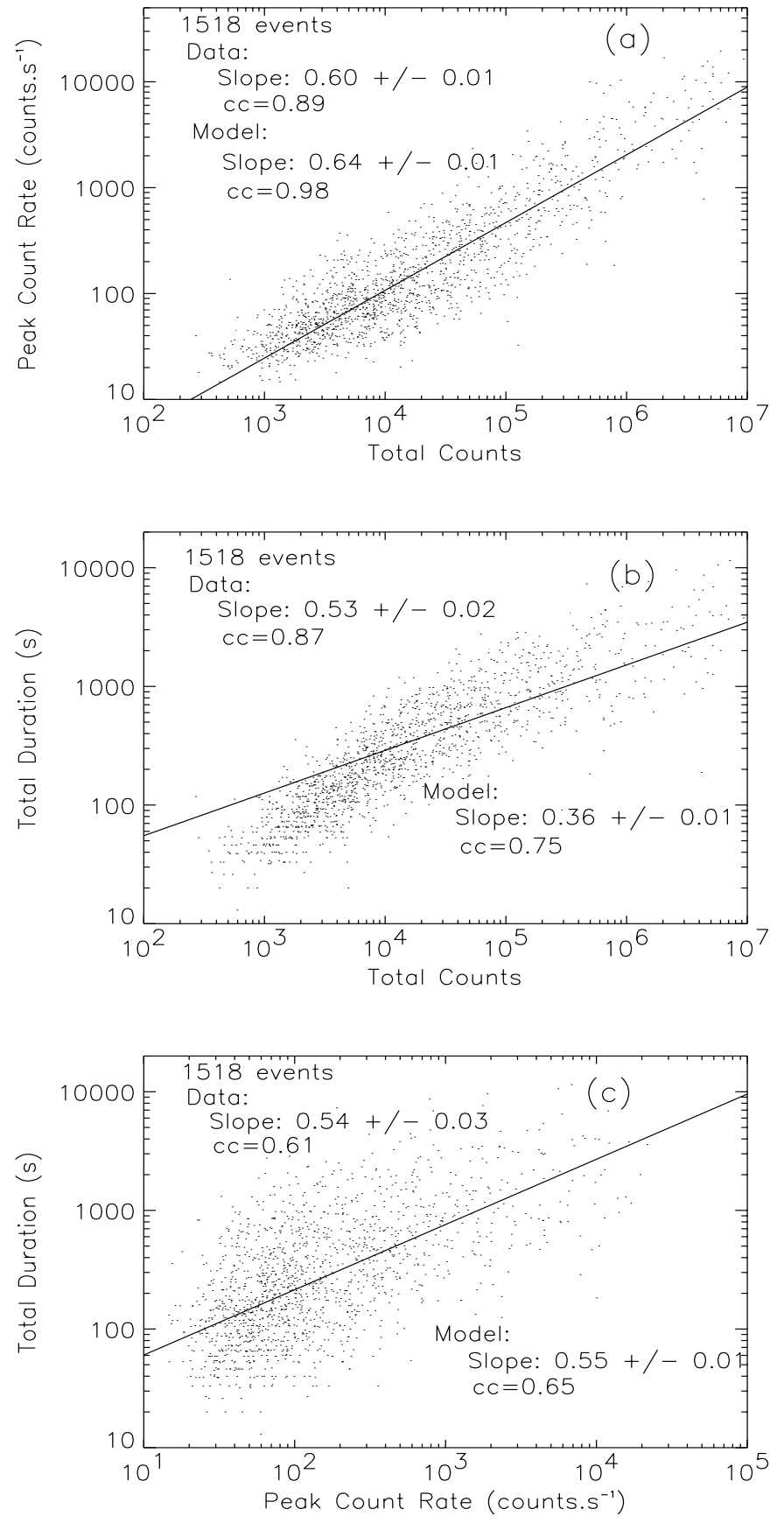

Fig. 5. a-c) Correlation scatter plots between the observed X-ray flare parameters. Data points correspond to WATCH events, while the fitting has been achieved by the respective scatter plots of the simulated events. Scatter plots refer to a) total counts vs. peak count rate, b) total counts vs. total duration and c) peak count rate vs. total duration

In Fig. 5 we show the correlation scatter plots between the different observed parameters of X-ray flares, where the points correspond to the WATCH data, while the fitting curves are the least-squares best-fit curves obtained for the correlation scatter plots of the associated model parameters. Normalization factors between observed and simulated values have been used for each fit by means of a maximum $\chi^{2}$ confidence-level method. 


\subsection{Correlations between successive events}

In Fig. 6 we investigate correlations between the peak luminosity of the simulated events vs. the elapsed time between successive events. In a former version of SOC models, such a test was performed by Lu et al. (1993) for the correlation between the total energy and the elapsed time and no correlation was found. Figure 6 a shows similar results concerning the peak luminosity and the elapsed time for the simulated events. In Fig. 6a we include the entire number of the simulated events, while in Fig. $6 \mathrm{~b}$ we include only the events belonging to the flat part of the peak luminosity distribution function (above $\sim 510^{4}$ arbitrary units). The calculated correlation coefficients are $-3.710^{-4}$ and -0.048 , respectively. The above result clearly indicates that the elapsed time between two successive events is not related to the events' sizes. This result stands in good agreement with similar results obtained from the observations.

A similar investigation is performed concerning the scatter plots of the peak luminosity vs. the distance between the locations of origin for successive events (Fig. 7). The distance is given by the length $|\boldsymbol{r}|\left(|\boldsymbol{r}|=\left|\boldsymbol{r}_{i}-\boldsymbol{r}_{i-1}\right|\right)$ between the position vectors $\boldsymbol{r}_{i-1}$ and $\boldsymbol{r}_{i}$ of the starting locations of two following events $i-1$ and $i$, respectively. Again, no correlation is found. It thus appears that an unstable configuration in the system may relax in a series of instabilities of any size that occur close to each other in space and time. The correlation coefficient for all the events (Fig. 7a) is $1.4910^{-3}$. A closer examination of Fig. 7a may lead to the conclusion that there are two populations of events (above and below a peak luminosity of $\sim 200$ ) showing analogous behavior. However, the events below a peak luminosity of $\sim 200$ correspond to the region of the distribution function which forms the low-energy cut-off. This interval cannot be fitted by power laws. The apparent division in Fig. 7a is, therefore, simply due to finite-resolution effects in the simulation grid. In Fig. 7b, a similar scatter plot of peak luminosity vs. distance of origin locations is shown for the hard part of the peak-count-rate distribution (Fig. 3b), revealing no correlation between the parameters (the correlation coefficient is 0.018). Unfortunately, we are unable to compare this result with the observations due to the lack of adequate spatial resolution in the WATCH experiment.

\section{Discussion and conclusions}

Comparisons between the statistical properties of observed X-ray bursts and the results of avalanche models have been so far performed in the works of $\mathrm{Lu}$ et al. (1993) and Georgoulis \& Vlahos (1998). The present work is another attempt to perform a comparison between the statistical properties of solar X-ray bursts observed by WATCH and the results of a modified cellular automaton Statistical Flare Model. The model relies on the assumption that solar active regions exhibit a self-organized critical behavior, with respect to externally induced
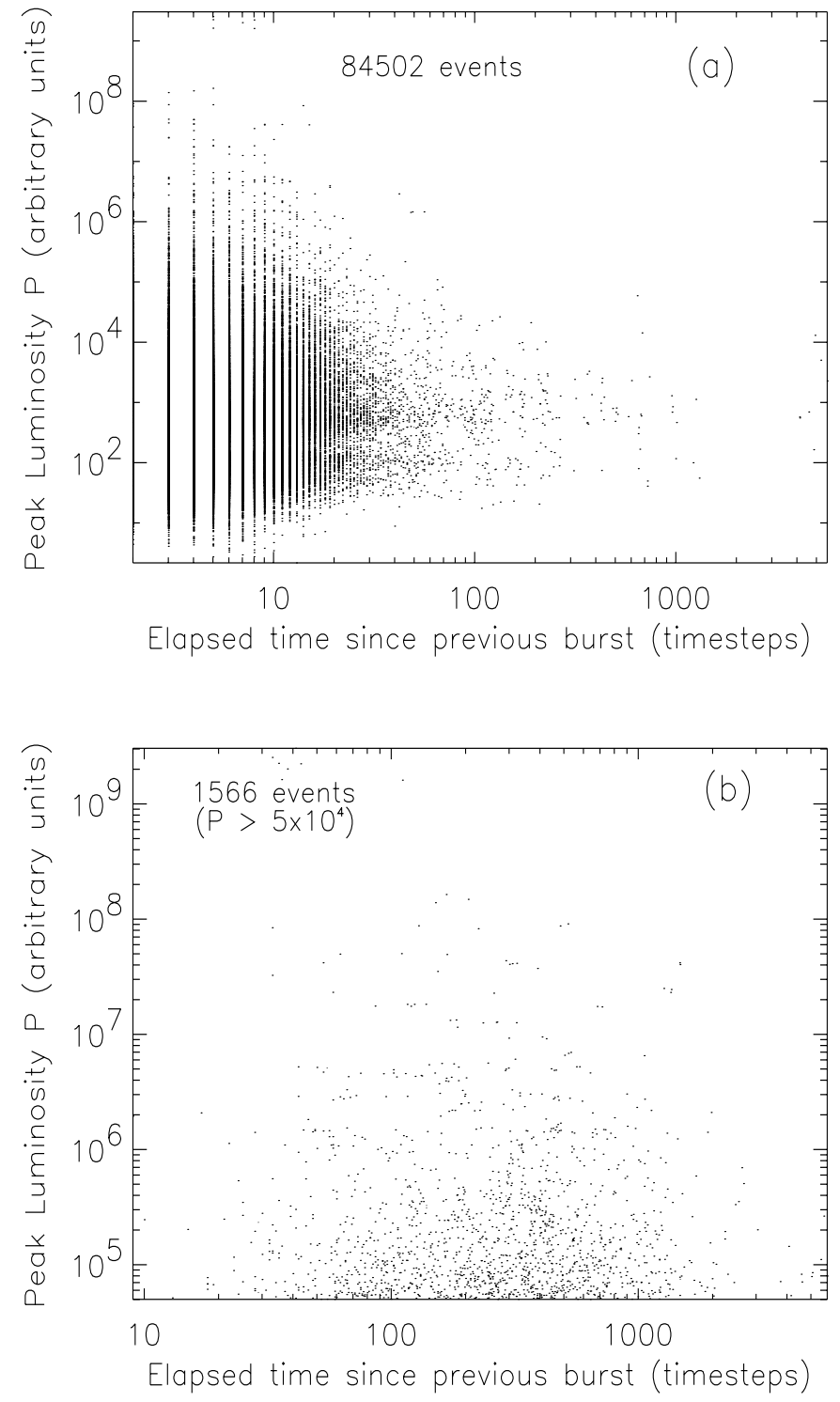

Fig. 6. a and b) Scatter plots of the elapsed time between successive events vs. the peak luminosity of simulated events. Scatter plots refer to a) the entire peak-luminosity distribution function and b) only the hard (flat) part of the distribution function of Fig. 3b

perturbations. A hypothetical critical threshold, assumed to be loosely related to a maximum current density in current layers may transform these perturbations into plasma instabilities that give rise to energy dissipation. Even in the absence of well-established physical links between the arbitrary model units and data units and even with the numerous warnings discussed in the following paragraphs, it is promising that one is able to derive similar powerlaw representations and similar slopes in the correlation scatter plots for the observations and the simulations by simply adjusting the external model driver.

Let us emphasize first that the Statistical Flare Model adopts a phenomenological approach to the problem of the dynamical evolution of active regions and flare energy release. The aim of the simulation is to show off the 

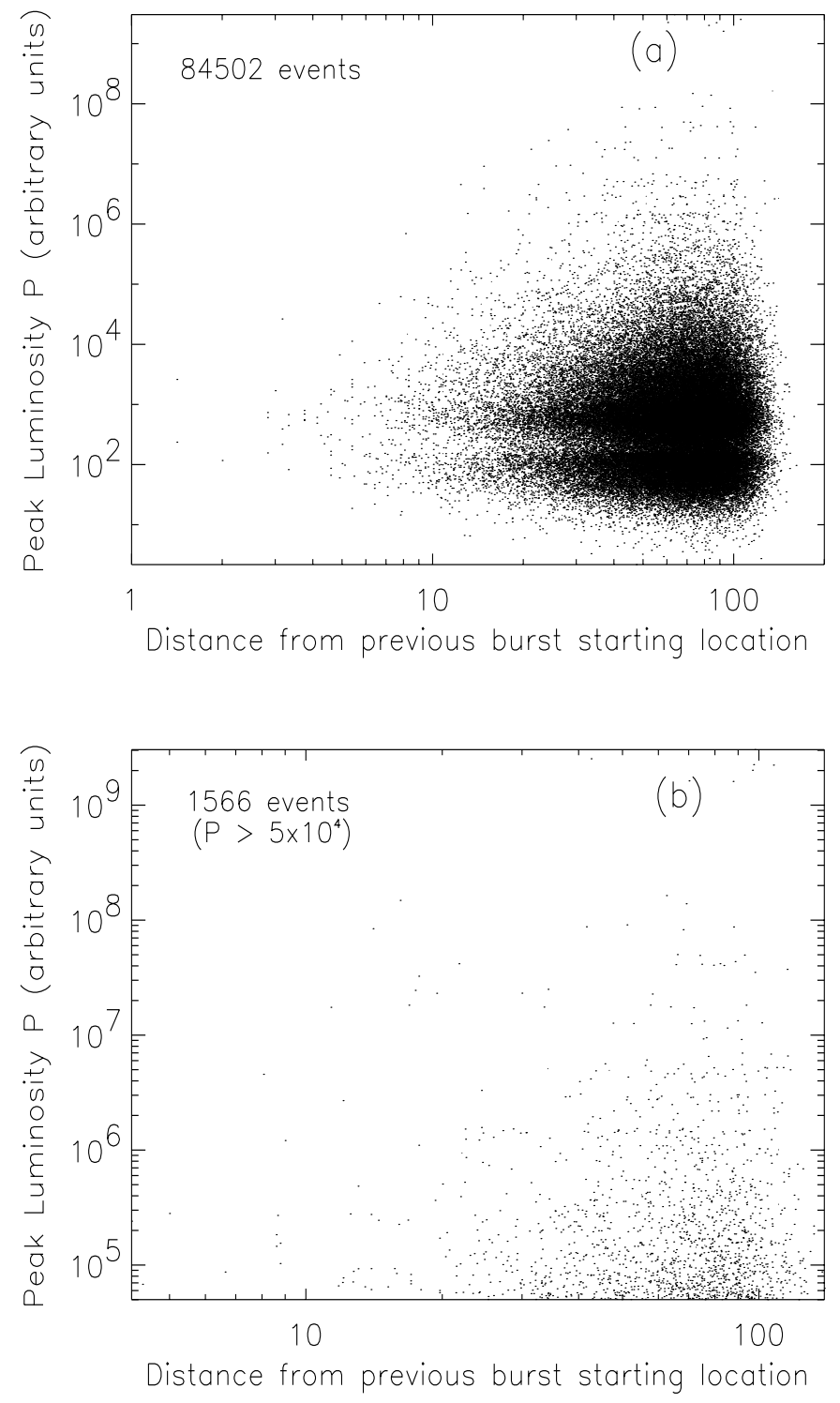

Fig. 7. a and b) Scatter plots of the distance between the starting locations for successive events vs. the peak luminosity of the simulated events. Scatter plots refer to a) the entire peak-luminosity distribution function and $\mathbf{b}$ ) only the hard (flat) part of the distribution function of Fig. 3b

main characteristics of the above-mentioned, inherently complex physical process. First, the external driver in the model restricts itself to the consequences of the actual, turbulent driver: the existing driver only deals with the change of magnetic stresses in localized magnetic configurations within active regions. A more comprehensive study lies within the framework of MHD (theory and simulations) and, moreover, deals with the study of the propagation of instabilities along the coronal magnetic field lines. Second, it is assumed that the excess of a critical threshold of current density automatically drives prominent, highly unstable current sheets enclosing micro-structures with dimensions of the order of the turbulent dissipative structures. In this sense, the localized magnetic Reynolds number is increased such as to allow energy dissipation through magnetic reconnection. The actual treatment of the process is, however, an outstanding problem of MHD Turbulence.

From the observational point of view, there is a number of additional warnings already mentioned in the introduction and in Sect. 4. The statistical study is based only on detector-dependent quantities, since photon spectral information is not available for the majority of the detected events. The study is performed on X-ray observations above $10 \mathrm{keV}$ in a photon energy range where thermal and non thermal radiation may be difficult to disentangle. In the absence of physical quantities, and also of a link between the arbitrary model units and physical units, the detector units have been associated with the model ones in a somewhat arbitrary, although reasonable, way. Finally, the conversion of the dissipated energy in thermal, or non-thermal, particle distributions is not included, as well as the radiation from these particles.

Having those open problems in mind, we proceeded with a purely statistical comparison between the results of the model and the observations. In Sect. 4.1 we compare the distribution functions of the parameters of the simulated and the observed events (total energy/total counts, peak luminosity/peak count rate and total duration in time-steps/seconds). The model exhibits a bimodal behavior in these distribution functions; this situation is not observed. The steeper population of weak events is indeed not detectable in the observed distributions and may correspond to yet un-observed, very weak energy-dissipation events. The comparison is thus based on the flat (hard) part of the simulated frequency distributions. The model also predicts that the resulting distribution functions may enclose different scaling indices; a feature which depends on the quantitative characteristics of the driver. This effect has been studied systematically in Georgoulis \& Vlahos (1998). Based on this work, the driver was suitably selected as to map the scaling index of the observational peak-count-rate distribution function (Fig. 1b). Remarkably, this selection automatically adjusts the other distribution functions to map observations closely. The agreement is excellent for total energy (Figs. 1a and 3a). However, in the case of the totalduration distribution functions (Fig. 1c), the agreement is not as perfect. Indeed, a double scaling behavior is found in the observations which is not reproduced by the simulation. While the slope of the steeper power law obtained for intermediate and large durations is closely consistent with the model's duration distribution function, the flat power law for the observed short-duration events is not reproduced by the model. This flat power law has not been obtained by other experiments, however, so it could be due to instrumental limitations or due to biases in the data selection process.

A second agreement between the statistical properties of the observations and of the model is also revealed when the overall data sets are filtered by some size criterion and divided into sub-sets. The aim of this test is to determine whether the overall distribution functions enclose 
component scaling behaviors. Filtering was accomplished either by dividing the total energies (total counts) of the simulated (observed) events in sub-sets with respect to different total-duration intervals or, inversely, by dividing the total durations of the observed and the simulated events in sub-sets with respect to different total-counts and total-energy intervals, respectively. The results, both in the observations and the model, indicate that different distribution functions are merged to form the final, overall distribution functions. It is clear in both observations and the simulation that filtering shorter (smaller) events reveals increasingly steeper power laws than the ones obtained for the bulk of events. The remarkable result is that sub-grouping maintains the power-law shape in the component distribution functions. From the statistical viewpoint, one could suggest that component power-law distribution functions are merged with different weighting factors and that large-size scales dominate when mingled with small-size scales. However, such an interpretation is not well-established. An inverse test of such a process was performed by Georgoulis \& Vlahos (1998) when different, well-formed power laws obtained for different driver indices were merged to produce an overall, well-formed power law.

In earlier observational studies (Bai 1993; Bromund et al. 1995), it has been reported that a distribution function of flare sizes (most commonly peak count rates or peak luminosities) may enclose a number of different scaling behaviors, whose individual features are lost in the overall, synoptic statistical treatment. The criteria for dividing the entire set of data values in subsets were then different, being based on observed periodicities of solar activity, but the results are similar. In the context of the present work, this effect is translated into obtaining steeper scaling laws for shorter or weaker events. This may imply that there is a trend of the power-law index to decrease as large events are excluded. It would be very interesting to see whether improved instrumental capabilities could reveal scaling laws significantly steeper for small-scale dissipation episodes, not necessarily detectable in X-ray wavelengths. This could provide a clue about the possible existence of nanoflaring activity (Parker 1988, 1989) and its statistical properties. From the observational point of view, hints for steeper logarithmic slopes in small-scale events have been reported for type I radio bursts (Mercier \& Trottet 1997) and EUV bright points (Krucker \& Benz 1998; Parnell \& Jupp 2000; Aletti et al. 2000). However, even in the observations, some controversy arises regarding the steepness of the distribution functions, either due to data selection and discrimination or due to different methods of deducing quantitative values from the observations (see, e.g. Berghmans et al. 1998; Aschwanden et al. 2000).

In Sect. 4.3 we investigated the correlations between parameters of the observed and the simulated events. Previous studies (Crosby et al. 1993; Lee et al. 1993; Bromund et al. 1995) performed on different X-ray databases reported significant correlations with moderateto-high correlation coefficients. This was also found in
WATCH data (Crosby 1996; Crosby et al. 1998b). In this paper, we investigate whether similar correlations can be found between the parameters of the simulated events. It is found that, within least-square absolute deviations, the slopes of the correlation scatter plots of observed parameters as well as the correlation coefficients are mostly reproduced. The most significant correlations for the observed/simulated events are found to link peak count rates/peak luminosities vs. total counts/total energies and total duration in seconds/time-steps vs. total counts/total energies. A fairly good agreement is found between the observations and the simulation for correlations between peak-count rate (peak luminosity) vs. total counts (total energy) and between total duration vs. peak count rate (peak luminosity). A larger discrepancy is found between the observations and the simulation for correlations between total duration vs. total counts (total energy). As already mentioned in Sect. 4.3, however, total duration is the observational parameter which is the less well defined and a number of factors such as particle transport/trapping and particle energy losses in the flaring medium are not considered in the Statistical Flare Model. These physical processes affect the temporal profiles of the events. This is probably the reason why the last scatter plot is the least well reproduced by the simulation. Furthermore, the conversion of the released energy into thermal/non-thermal particle distribution should also be incorporated into the model for a better comparison with observations.

No correlation was found between the elapsed time interval between two successive X-ray bursts coming from the same active region and the peak intensity of the latest burst (Crosby 1996; Crosby et al. 1998b). Moreover, the distribution function of the elapsed times was found to obey an exponential law. The same situation arises with the events simulated by the Statistical Flare Model, either for the entire total-energy distribution function, or for its hard part alone (Sect. 4.4). The random nature of the triggering process in the cellular automaton gives rise to purely Poissonian statistics in the distribution function of the elapsed times. We applied a further test with the model results, investigating correlations linking the distances of origin locations between successive events and the total energies of events. The result shows that these parameters are not correlated, as well. Although beyond comparison with WATCH data, this model prediction could potentially be tested with future spatially resolved observations of hard X-ray bursts originating from a given active region.

The absence of spatio-temporal correlations in the simulation and temporal correlations in the observations, implies that unstable topologies do not tend to release their stored magnetic energy in one event. Instead, they may well return to a "ground energy state" by means of a series of successive events of any size. In the Statistical Flare Model, this feature is embedded in the relaxation rules. It is also due to the highly variable, non-linear driver and the random selection of the cells to be perturbed. 
These algorithmic rules suitably fit to the statistics of the observed data. In the real system, the statistical behavior of the energy release process may be similar. In view of the profound complexity and the fully developed turbulent state built in active regions, it is likely that the nonpotential energy stored in a magnetic topology leading to energy release is not distributed smoothly in a single current sheet, but it is fragmented and enveloped into an ensemble of current sheets of any size, distributed randomly in space. Current sheets may also be disrupted randomly in time, according to the local conditions. It is worth noting that existing two- or three-dimensional MHD models are strongly in favor of this complexity scenario (Biskamp \& Welter 1989; Mikic et al. 1989; Longcope \& Sudan 1994; Galsgaard \& Nordlund 1996; Georgoulis et al. 1998).

In conclusion, the present work shows that cellular automata SOC models are able to reproduce efficiently most of the statistical properties of solar flares, at least those seen in hard X-ray wavelengths (Lu et al. 1993; Georgoulis \& Vlahos 1998 and this work). However, it is clear that a more physical approach to be compared with observations, requires substantially refined cellular automata SOC models. A first, important step would be to establish a more physical connection between the cellular automata evolution rules and fundamental laws of MHD. Attempts to link the two approaches are under way (Vassiliadis et al. 1998; Isliker et al. 1998, 2000a,b). The discrete evolution rules of the model would be then controlled by the physics of the actual system and, moreover, the association of the arbitrary model units with physical units would become a tractable task. The assumption of an intrinsic self-organization built in coronal magnetic structures with respect to some critical-parameter values whose excess means magnetic reconnection and diffusion of magnetic field lines, should be thoroughly investigated. Once the validity of SOC (or, at least, SOC-type) cellular automata models is established on a physical basis, it would provide an approach complementary to MHD simulations, aiming to a deeper understanding of the dynamical evolution of solar active regions and the energy release process in solar flares.

Acknowledgements. We are grateful to Prof. Loukas Vlahos for many illuminating discussions and various suggestions. We also thank Dr. Heinz Isliker for numerous comments and suggestions in the text. One of us (MKG) wishes to acknowledge the support of the Greek State Foundation for Scholarships (IKY) to the accomplishment of this work.

\section{References}

Aletti, V., Velli, M., Bocchialini, K., Georgoulis, M., \& Vial, J.-C. 2000, ApJ, 544, 550

Aschwanden, M. J., Tarbell, T. D., Nightingale, R. W., Scrijver, C. J., \& Title, A. 2000, ApJ, 535, 1047

Bai, T., \& Sturrock, P. 1993, ApJ, 409, 476

Bai, T. 1993, ApJ, 404, 805

Bak, P., Tang, C., \& Wiesenfield, K. 1987, Phys. Rev. Lett., 59 , No. 4,381
Bak, P., Tang, C., \& Wiesenfield, K. 1988, Phys. Rev. A., 38, No. 1, 364

Berghmans, D., Clette, F., \& Moses, D. 1998, A\&A, 36, 1039

Biesecker, D. A. 1994, Ph.D. Thesis, University of New Hampshire

Biskamp, D., \& Welter, H. 1989, Phys. Fluids, B1, 1964

Bromund, K. R., McTiernan, J. M., \& Kane, S. R. 1995, ApJ, 455, 733

Crosby, N. B., Aschwanden, M. J., \& Dennis, B. R. 1993, Sol. Phys., 143, 275

Crosby, N. B. 1996, Ph.D. Thesis, Université Paris VII, France

Crosby, N. B., Lund, N., Vilmer, N., \& Sunyaev, R. 1998a, A\&AS, 130, 233

Crosby, N. B., Vilmer, N., Lund, N., \& Sunyaev, R. 1998b, A\&A, 334, 299

Datlowe, D. W., Elcan, M. J., \& Hudson, H. S. 1974, Sol. Phys., 39, 155

Dennis, B. R. 1985, Sol. Phys., 100, 465

Galsgaard, K. 1996, A\&A, 315, 312

Galsgaard, K., \& Nordlund, A. 1996, J. Geophys. Res., 101, 13445

Georgoulis, M., Kluiving, R., \& Vlahos, L. 1995, Phys. A, 218, 191

Georgoulis, M. K., \& Vlahos, L. 1996, ApJ, 469, L135

Georgoulis, M. K., \& Vlahos, L. 1998, A\&A, 336, 721

Georgoulis, M. K., Velli, M., \& Einaudi, G. 1998, ApJ, 497, 957

Hudson, H. S. 1991, Sol. Phys., 133, 357

Isliker, H., Anastasiadis, A., Vassiliadis, D., \& Vlahos, L. 1998, A\&A, 335, 1085

Isliker, H., Anastasiadis, A., \& Vlahos, L. 2000a, A\&A, 363, 1134

Isliker, H., Anastasiadis, A., \& Vlahos, L. 2000b, A\&A, submitted

Kadanoff, L. P., Nagel, S. R., Wu, L., \& Zhou, S.-M. 1989, Phys. Rev. A, 39, 6524

Krucker, S., \& Benz, A. O. 1998, ApJ, 501, L213

Lee, T. T., Petrosian, V., \& McTiernan, M. 1993, ApJ, 412, 401

Lin, R. P., Schwartz, R. A., Kane, S. R., Pelling, R. M., \& Hurly, K. C. 1984, ApJ, 283, 421

Longcope, L., \& Sudan, L. 1994, ApJ, 437, 491

Lu, E. T., \& Hamilton, R. J. 1991, ApJ, 380, L89

Lu, E. T., Hamilton, R. J., McTiernan, J. M., \& Bromund, K. R. 1993, ApJ, 412, 841

Mercier, C., \& Trottet, G. 1997, ApJ, 474, L65

Mikic, Z., Schnack, D. D., \& Van Hoven, G. 1989, ApJ, 338, 1148

Parker, E. N. 1988, ApJ, 330, 474

Parker, E. N. 1989, Sol. Phys., 121, 271

Parnell, C. E., \& Jupp, P. E. 2000, ApJ, 529, 554

Pearce, J., Rowe, A. K., \& Yeung, J. 1993, Ap\&SS, 208, 99

Rieger, E., Share, G. H., Forrest, D. J., et al. 1984, Nature, 312,623

Sturrock, P. A., \& Bai, T. 1992, ApJ, 397, 337

Trottet, G., \& Vilmer, N. 1997, in The Production of FlareAccelerated Particles at the Sun, ed. G. M. Simnett, C. E. Allisandrakis, \& L. Vlahos, Proc. 8th European Meeting on Solar Physics, Solar and Heliospheric Plasma Physics, LNP, 489, 219

Vassiliadis, D., Anastasiadis, A., Vlahos, L., \& Georgoulis, M. 1998, ApJ, 509, L53

Vlahos, L., Georgoulis, M., Kluiving, R., \& Paschos, P. 1995, A\&A, 299, 897 